\title{
Tratamento de crescimento epitelial persistente pós-Lasik com debridamento mecânico, uso de álcool a $20 \%$ e cola de fibrina
}

Treatment of persistent epithelial ingrowth after Lasik with mechanical debridement, use of $20 \%$ alcohol and fibrin glue

Marina Alves Lucena' (), Ermano de Melo Alves ${ }^{2}$ (1), Henrique Brandt Krause ${ }^{1}$ (1), Márcio Martins de Melo Alves ${ }^{3}$ (D) Pedro Lukas do Rêgo Aquino ${ }^{4}$ (i)

${ }^{1}$ Centro Universitário Maurício de Nassau, Recife, PE, Brasil. ${ }^{2}$ Oftalmax Hospital de Olhos, Recife, PE, Brasil.

${ }^{3}$ Faculdade Pernambucana de Saúde, Recife, PE, Brasil.

${ }^{4}$ Universidade de Pernambuco, Recife, PE, Brasil.

Lucena MA, Alves EM, Krause HB, Alves MM, Aquino PL. Tratamento de crescimento epitelial persistente como citar: debridamento mecânico, uso de álcool a 20\% e cola de fibrina. Rev Bras Oftalmol. 2021;80(4):e0020.

Descritores:

Crescimento epitelial; Álcool; Cola de fibrina; Ceratomileuse assistida por excimer laser in situ; Acuidade visual

Keywords:

Epithelial ingrowth; Alcohol;

Fibrin glue; Laser in situ keratomileusis; Visual acuity

Recebido: 17/05/2020

\section{Aceito:}

$15 / 4 / 2021$

Autor correspondente: Marina Alves Lucena

Rua Caio Pereira, 175 - Rosarinho CEP. 52041-045 - Recife, PE, Brasil E-mail: marinaalveslucena@gmail.com

Instituição: Oftalmax Hospital de Olhos.

Fonte de auxílio à pesquisa: não financiado.

Conflitos de interesse: os autores declaram que não há conflitos de interesses.

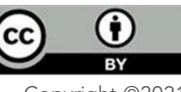

Copyright (C2021

\section{RESUMO}

O Lasik é a técnica de cirurgia refrativa mais utilizada no mundo. Apesar de segura e efetiva, ela pode levar a algumas complicações. O crescimento epitelial pós-Lasik é uma complicação pós-operatória incomum, com prevalência maior em casos de retratamento. Geralmente, é um achado não progressivo e assintomático, que não requer tratamento, mas, em uma minoria de pacientes, os sintomas podem ser clinicamente significantes e variados. O tratamento é feito com debridamento mecânico do crescimento epitelial, mas alguns recursos adjuvantes também podem ser utilizados. O presente estudo consiste em um relato de caso de paciente com crescimento epitelial pós-Lasik que apresentou quatro recidivas após intervenções de debridamento epitelial, sutura de lamela corneana e ablação a laser. No quinto procedimento, o paciente foi finalmente tratado com combinação de debridamento epitelial, uso de álcool a 20\% e cola de fibrina. Entretanto, a regressão do crescimento epitelial e a melhora da acuidade visual só ocorreram ao longo dos meses após a intervenção, o que mostra a importância de esperar um tempo para que ocorra a melhora da visão no pós-operatório, evitando-se reintervenções.

\section{ABSTRACT}

Lasik is the most often performed laser refractive surgery worldwide. Despite its efficacy and safety, some complications may occur. Epithelial ingrowth is a rare postoperative complication of Lasik, with an increased prevalence in cases of retreatment. Epithelial ingrowth is usually a nonprogressive and asymptomatic finding, which requires no treatment; however, in a minority of cases, symptoms may be clinically significant and diverse. Treatment is done with mechanical debridement of the affected interface, and additional interventions may be required. This study reported a case of recalcitrant epithelial ingrowth after Lasik, whichrelapsed four times after mechanical debridement, flap lift and laser ablation. In the fifth intervention, the patient was finally treated with a combined scraping/use of $20 \%$ alcohol and fibrin glue. However, regression of epithelial ingrowth and better visual acuity were only observed some months after the intervention, which shows the importance of waiting for better vision in the postoperative period, thus avoiding new reinterventions. 


\section{INTRODUÇÃO}

O laser in situ keratomileusis (Lasik) é a técnica de cirurgia refrativa mais utilizada no mundo e consiste na confecção de um retalho corneano, seguida da fotoablação estromal com excimer laser. Apesar de ser uma técnica segura e efetiva, a criação de uma interface entre a lamela corneana e o leito estromal pode levar a complicações, como ceratite infecciosa, ceratite lamelar difusa e crescimento epitelial pós-Lasik. ${ }^{(1,2)}$

O crescimento de células epiteliais entre a lamela e o leito estromal é uma complicação pós-operatória incomum, com prevalência que varia de zero a 3,9\%, em casos de Lasik primário, e de 10\% a 20\% em casos de retratamento. ${ }^{(3)} \mathrm{Na}$ maioria das vezes, o crescimento epitelial é um achado não progressivo e assintomático que não requer tratamento. Entretanto, em uma minoria de pacientes $(0,92 \%),{ }^{(4)}$ os sintomas podem ser clinicamente significativos e incluem redução da acuidade visual, ofuscamento, irregularidades e danos à superfície da córnea, o que leva necessariamente à reintervenção cirúrgica.

O tratamento inicial do crescimento epitelial envolve levantamento da lamela corneana com debridamento mecânico do crescimento epitelial, seguido de cuidadosa reposição da lamela, entretanto, a taxa de recorrência desse procedimento pode alcançar $44 \% .{ }^{(5)}$ Para melhorar a efetividade, alguns recursos adjuvantes podem ser utilizados para garantir a eliminação de células epiteliais residuais e facilitar a adesão da lamela. Sutura da lamela, uso de cola de fibrina, etanol, mitomicina C, YAG laser e realização de uma ablação a laser phototerapeutic keratectomy (PTK) são recursos adicionais aos casos recalcitrantes. ${ }^{(4)}$

Descrevemos o relato do caso de um paciente com crescimento epitelial pós-Lasik, que apresentou quatro recidivas após intervenções de debridamento epitelial, sutura de lamela e ablação a laser. No quinto procedimento, o paciente foi finalmente tratado com combinação de debridamento epitelial, uso de álcool a 20\% e cola de fibrina. Entretanto, a regressão do crescimento epitelial e a melhora da acuidade visual só ocorreram ao longo dos meses após a intervenção, o que mostra a importância de esperar um tempo para que se alcance a melhora da visão no pós-operatório, evitando-se reintervenções.

\section{RELATO DO CASO}

Paciente de 62 anos submetido a Lasik hipermetrópico chegou ao nosso serviço com queixa de baixa visual em olho direito (OD). Relatou já ter sido submetido a três cirurgias nesse olho, sem melhora da visão. Ao exame, constatamos crescimento epitelial extenso em ambos os olhos e sutura em bordo inferonasal em OD. Sua acuidade visual e refração em abril de 2016 era de +4,00-3,00x95 (20/40) para o OD e +0,50-0,50x80 (20/30) para o olho esquerdo (OE). Foi realizado, então, no mesmo mês, um novo levantamento da lamela em ambos os olhos, com debridamento epitelial no leito estromal, na face posterior e na margem da lamela, além de ablação de $10 \mu \mathrm{m}$ com excimer laser (PTK) WaveLight ${ }^{\circledR}$ EX500 Alcon, mais irrigação intensa nas faces anterior e posterior do flap, secagem com esponja Meroce ${ }^{\circledR}$ para garantir boa aderência do flap e lente de contato terapêutica no final. No pós-operatório, utilizamos uma combinação de colírio antibiótico com corticoide (moxifloxacino e dexametasona) quatro vezes ao dia por 1 semana e lubrificação artificial com carmelose sódica cinco vezes ao dia. Em maio de 2016, o OD apresentava acuidade visual de 20/40 com a refração +1,50-1,50 x90, e o OE alcançava 20/25 com $+0,50$ esférico.

Em junho de 2016, um novo crescimento epitelial foi observado em ambos os olhos, porém, como não comprometia a acuidade visual (AV) de maneira significativa (sem correção, 20/40), optamos por observar. Em março de 2017, o paciente retornou com relato de piora na visão em $\mathrm{OD}(\mathrm{AV}=+1,00,20 / 70)$ (Figura 1). O OE manteve-se estável, com boa AV (20/30 sem correção e 20/25 com +0,50). Decidimos por uma nova intervenção em OD. Realizamos um novo debridamento do estroma e da face posterior da lamela corneana, aplicação de álcool a 20\% no estroma, irrigação copiosa e, posteriormente, cola de fibrina na margem da lamela $360^{\circ} \mathrm{com}$ lente de contato terapêutica no final (Figuras 2 e 3) com o mesmo esquema terapêutico pós-operatório do primeiro procedimento. No segundo mês de pós-operatório, em maio de 2017, não havia crescimento epitelial em nenhum dos olhos e a AV corrigida em OD era 20/40 e 20/25 em OE; atribuímos a baixa visão em OD à possível irregularidade topográfica, porém optamos por não realizar nenhum exame de imagem nessa data. Prescrevemos os óculos $(\mathrm{OD}=+1,75-2,00 \mathrm{X} 100$ e $\mathrm{OE}=+0,75$ $-0,75 X 115)$ e solicitamos nova avaliação mais completa em 3 meses, com uso contínuo de lubrificantes.

O paciente só retornou após 19 meses da segunda intervenção, em janeiro de 2018, e apresentava um novo crescimento epitelial em OD no mesmo local inicial (inferonasal), porém menor e sem atingir eixo visual, com piora da visão (AV com correção = 20/60). Optamos por não mais intervir cirurgicamente, pois o próprio paciente já apresentava restrições para um novo procedimento. Foi esclarecida a necessidade obrigatória de uma nova cirurgia, caso houvesse aumento significativo do crescimento 


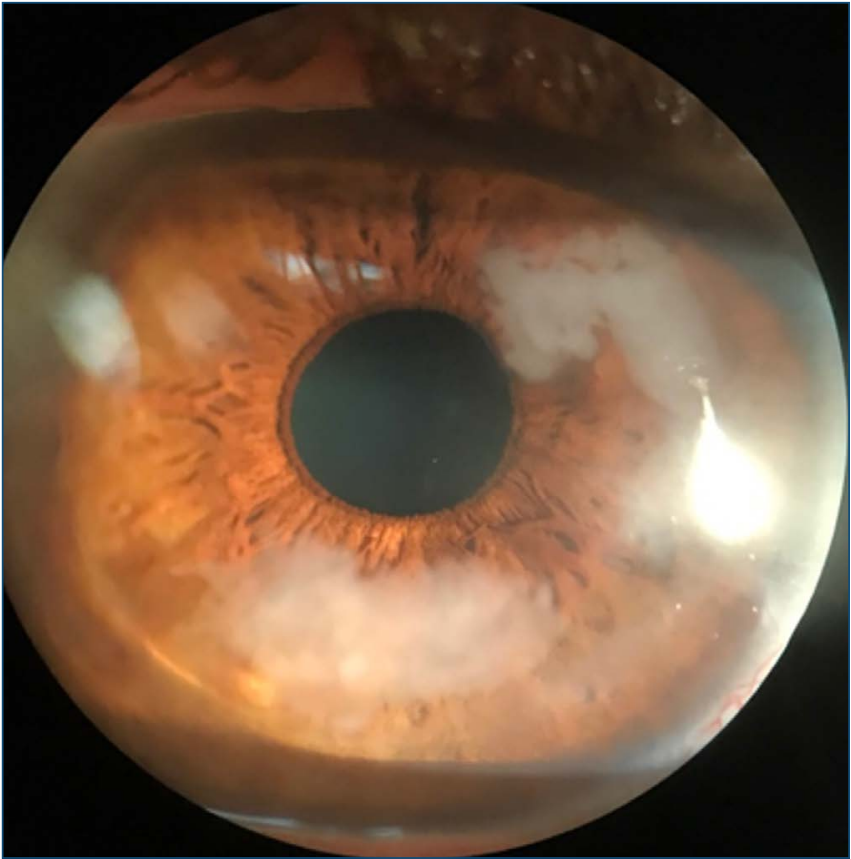

Figura 1. Crescimento epitelial em olho direito. Foto de março de 2017.

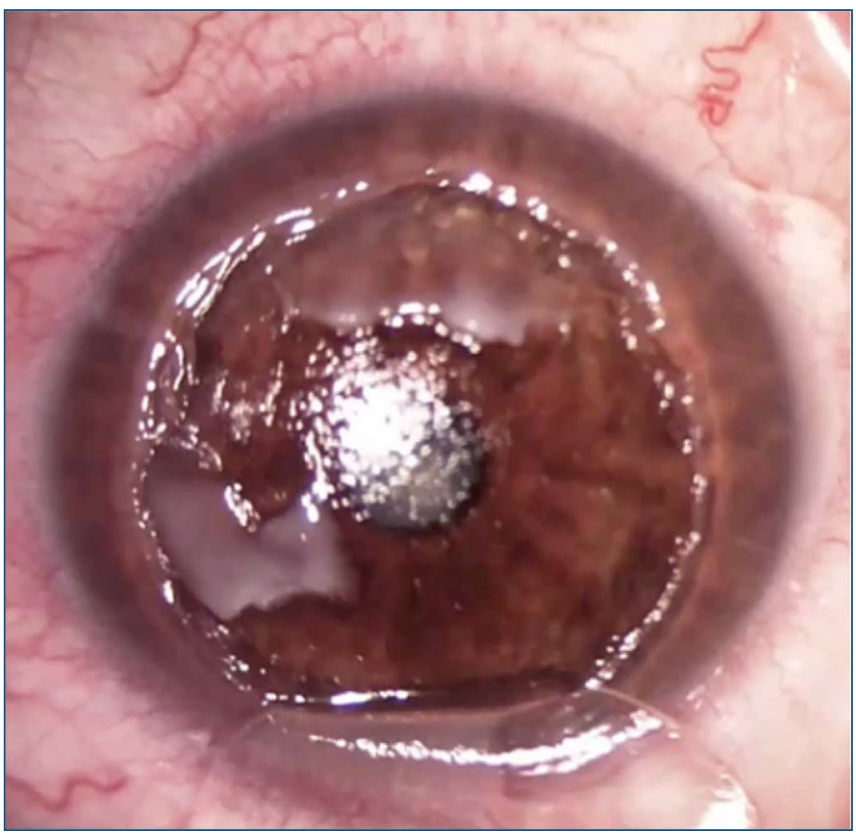

Figura 2. Crescimento epitelial em leito estromal do olho direito após levantamento da lamela corneana durante segunda intervenção em nosso serviço. Foto de março de 2017.

epitelial ou melting do flap. Durante o seguimento, observamos mudanças refracionais e da AV ao longo dos meses: após 25 meses (+1,50 e 20/60) e após 29 meses (-0,50 e 20/50). Na sequência, o paciente se ausentou por um longo período, só retornando 1 ano depois, em novembro de 2019, com regressão total do crescimento epitelial (Figura 4). A refração em OD era $+1,00-1,00 \times 100$ (20/40) e no OE era +1,00-1,00x110 (20/20).

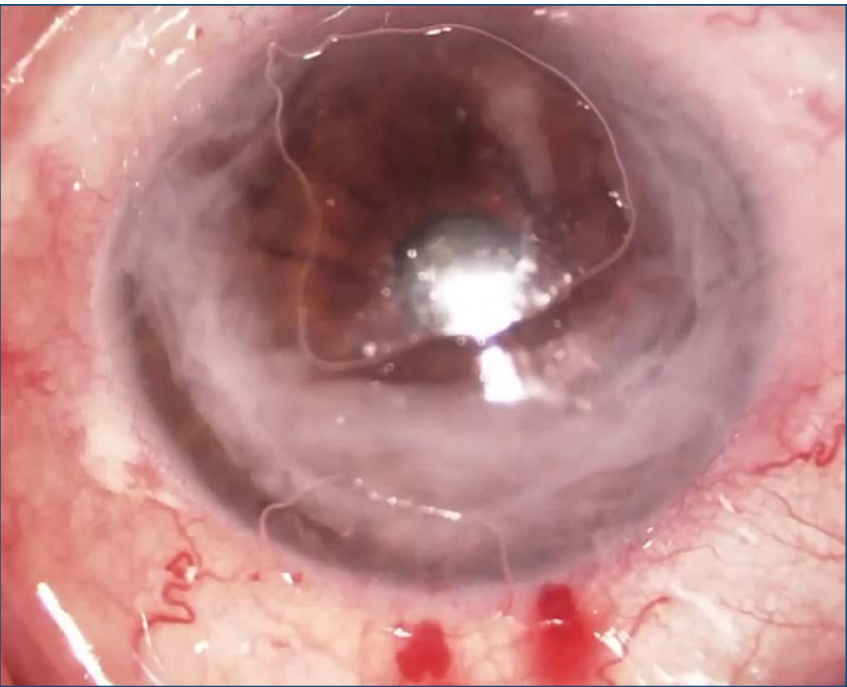

Figura 3. Resultado do olho direito após a realização da segunda intervenção cirúrgica em nosso serviço. Foto de março de 2017.

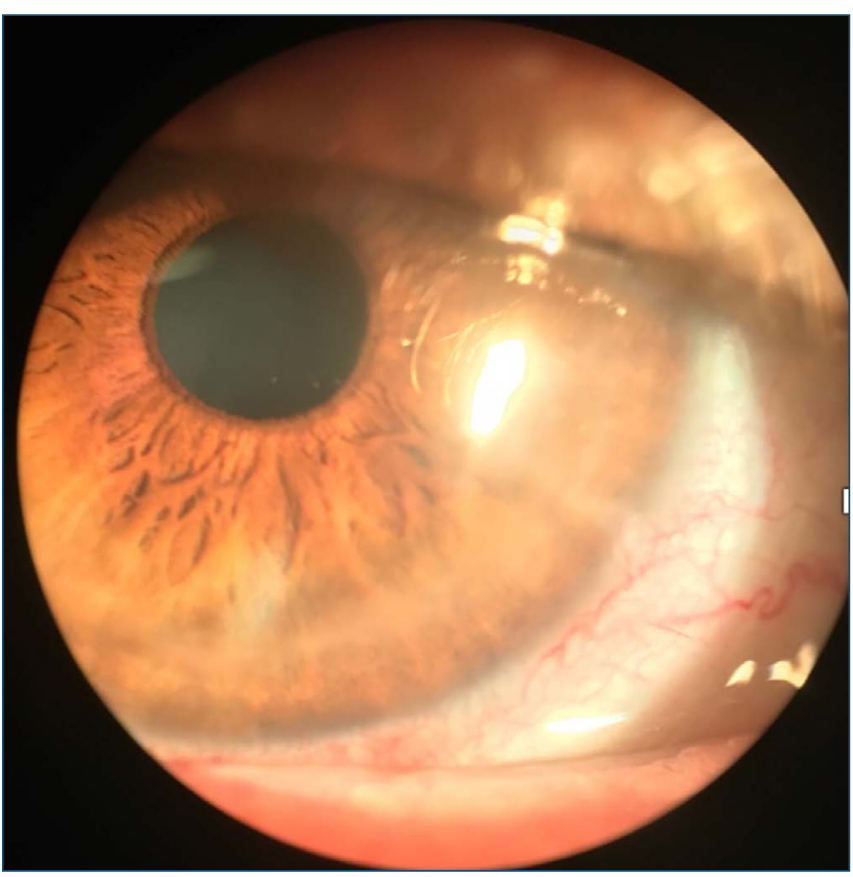

Figura 4. Regressão total do crescimento epitelial. Foto de novembro de 2019.

\section{DISCUSSÃO}

Friehman at al., ${ }^{(6)}$ após analisarem retrospectivamente mais de 30 mil olhos submetidos ao Lasik, descreveram os principais fatores de risco para crescimento epitelial: levantamento da lamela corneana, tempo prolongado entre o Lasik primário e o retratamento (pico entre 4 e 5 anos), zona óptica pequena, moderada a severa hipermetropia, ablação profunda e cirurgião com baixo volume cirúrgico. Identificamos pelo menos dois fatores de risco em nosso paciente: o levantamento da lamela e o tratamento hipermetrópico. Os demais fatores, não pudemos confirmar ou descartar, pela ausência de dados clínicos. 
O levantamento da lamela para uma nova ablação aumenta a possibilidade da criação de uma fístula entre o leito estromal e a superfície ocular, facilitando a migração de células epiteliais. Essa hipótese, levantada por Wang et al.,(1) parece-nos mais provável do que a simples proliferação de células epiteliais residuais, visto que, em nosso paciente, a área inicial das recidivas era sempre a mesma (nasal inferior) e, durante a segunda cirurgia, era evidente uma lesão estromal na margem (Figura 5).

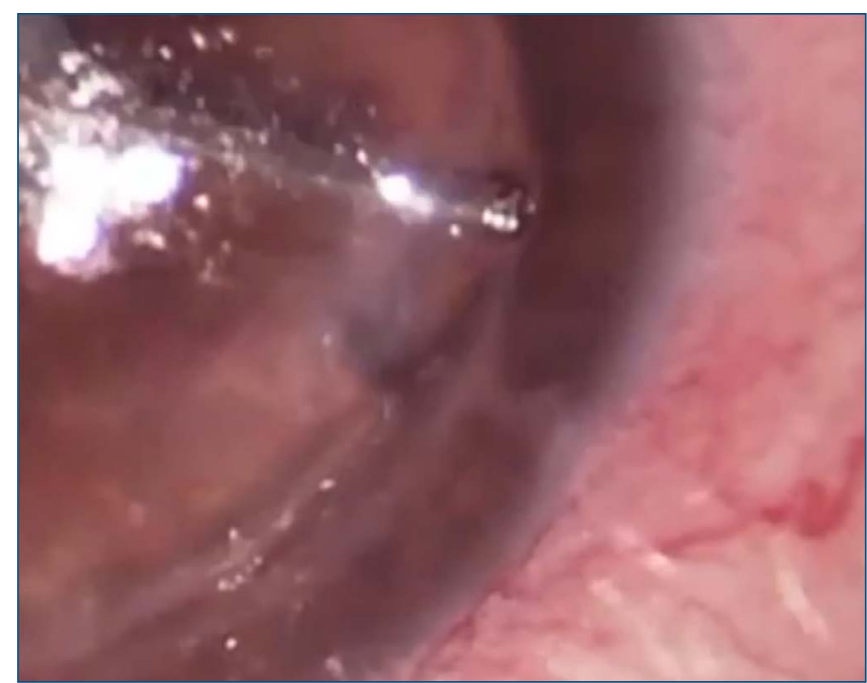

Figura 5. Lesão na margem do leito estromal visualizada durante a segunda intervenção em nosso serviço. Foto de março de 2017.

O tratamento hipermetrópico produz maior ablação periférica em relação à ablação miópica, ultrapassando, algumas vezes, a margem do leito estromal, dificultando o perfeito encaixe da lamela e reduzindo a aderência entre as bordas da lamela e o estroma. Essa assimetria facilita a passagem de células epiteliais em áreas danificadas ou irregulares, principalmente após o uso de instrumentos cirúrgicos para levantar uma lamela previamente bem posicionada. ${ }^{(7)}$

A possibilidade de existência de uma fístula pode explicar o insucesso da nossa primeira intervenção - o debridamento epitelial associado à ablação com excimer laser (PTK) - pois o objetivo, nesse caso, era eliminar células residuais no estroma, e não tinha nenhum efeito terapêutico em uma fístula preexistente. Reforçando essa hipótese, 2 meses após nossa intervenção, o paciente apresentou novo crescimento epitelial com comprometimento significativo da acuidade visual.

Na segunda intervenção feita em nosso serviço, utilizamos álcool a 20\% e cola de fibrina como terapias adjuvantes. O uso do álcool nesse tipo de cirurgia objetiva desvitalizar eventuais células epiteliais residuais que permaneceram na interface. ${ }^{(4)}$ Já a cola é utilizada para promover melhor aderência da lamela ao leito estromal e inibir o acesso de células epiteliais à interface por meio da provável fístula (nossa principal hipótese). Em conjunto, essa opção foi efetiva na resolução do nosso caso, apesar do longo tempo percorrido até a completa resolução.

A remissão espontânea do último episódio de crescimento epitelial em nosso caso foi surpreendente, mas não é inédita na literatura. Em 2005, Lin et al. ${ }^{\left({ }^{(8)}\right.}$ descreveram um caso com regressão após 20 meses de acompanhamento e, em 2013, Zhang et al.(9) publicaram relato semelhante após 6 meses de observação sem intervenção cirúrgica.

Concluímos então que, se tivéssemos valorizado inicialmente a possibilidade de uma fístula, poderíamos ter optado pelo debridamento com álcool a 20\% e cola de fibrina já no primeiro procedimento, evitando o uso do excimer laser e uma intervenção a mais. O tempo até a completa resolução também deve ser enfatizado: foi necessário mais de 1 ano para a remissão total do crescimento epitelial e a melhora significativa da acuidade visual com estabilidade refracional.

Este relato de caso sugere, portanto, que o uso de cola de fibrina na margem do flap em casos recorrentes pode ser feito precocemente para evitar novos episódios de crescimento epitelial, e um tempo de observação longo é aconselhável, antes de realizar novas intervenções em casos menos graves.

\section{REFERÊNCIAS}

1. Wang MY, Maloney RK. Epithelial ingrowth after laser in situ keratomileusis. Am J Ophthalmol. 2000;129(6):746-51.

2. Lin RT, Maloney RK. Flap complications associated with lamellar refractive surgery. Am J Ophthalmol. 1999;127(2):129-36.

3. Ting DS, Srinivasan S, Danjoux JP. Epithelial ingrowth following laser in situ keratomileusis (LASIK): prevalence, risk factors, management and visual outcomes. BMJ Open Ophthalmol. 2018;3(1):e000133.

4. Wilde C, Messina M, Dua HS. Management of recurrent epithelial ingrowth following laser in situ keratomileusis with mechanical debridement, alcohol, mitomycin-C, and fibrin glue. J Cataract Refract Surg. 2017;43(7):980-4.

5. Rojas MC, Lumba JD, Manche EE. Treatment of epithelial ingrowth after laser in situ keratomileusis with mechanical debridement and flap suturing. Arch Ophthalmol. 2004;122(7):997-1001.

6. Friehmann A, Mimouni M, Nemet AY, Sela T, Munzer G, Kaiserman I. Risk Factors for Epithelial Ingrowth Following Microkeratome-Assisted LASIK. J Refract Surg. 2018;34(2):100-5.

7. Mohamed TA, Hoffman RS, Fine $\mathrm{IH}$, Packer M. Post-laser assisted in situ keratomileusis epithelial ingrowth and its relation to pretreatment refractive error. Cornea. 2011;30(5):550-2.

8. Lin JM, Tsai YY, Tseng SH. Spontaneous regression of dense epithelial ingrowth after laser in situ keratomileusis. J Refract Surg. 2005;21(3):300-2.

9. Zhang R, Jhanji V, Sun L, Zhang M. Spontaneous resolution of delayed epithelial ingrowth after LASIK. Eye Contact Lens. 2013;39(6):400-1. 